\title{
MEASUREMENTS AND MODELING OF TOTAL SOLAR IRRADIANCE IN X-CLASS SOLAR FLARES
}

\author{
Christopher Samuel Moore ${ }^{1,2,3}$, Phillip Clyde Chamberlin ${ }^{4}$, and Rachel Hock ${ }^{5}$ \\ ${ }^{1}$ Center for Astrophysics and Space Astronomy, University of Colorado, UCB 389, Boulder, CO 80309, USA \\ ${ }^{2}$ Laboratory for Atmospheric and Space Physics, University of Colorado, UCB 600, Boulder, CO 80303, USA \\ ${ }^{3}$ Astrophysical and Planetary Sciences Department, University of Colorado, UCB 391, Boulder, CO 80309, USA \\ ${ }^{4}$ Solar Physics Laboratory, NASA Goddard Space Flight Center, Greenbelt, MD 20771, USA \\ ${ }^{5}$ Space Vehicle Directorate, Air Force Research Laboratory, Kirtland Air Force Base, NM 87117, USA \\ Received 2013 October 23; accepted 2014 March 26; published 2014 May 1
}

\begin{abstract}
The Total Irradiance Monitor (TIM) from NASA's SOlar Radiation and Climate Experiment can detect changes in the total solar irradiance (TSI) to a precision of $2 \mathrm{ppm}$, allowing observations of variations due to the largest X-class solar flares for the first time. Presented here is a robust algorithm for determining the radiative output in the TIM TSI measurements, in both the impulsive and gradual phases, for the four solar flares presented in Woods et al., as well as an additional flare measured on 2006 December 6. The radiative outputs for both phases of these five flares are then compared to the vacuum ultraviolet (VUV) irradiance output from the Flare Irradiance Spectral Model (FISM) in order to derive an empirical relationship between the FISM VUV model and the TIM TSI data output to estimate the TSI radiative output for eight other X-class flares. This model provides the basis for the bolometric energy estimates for the solar flares analyzed in the Emslie et al. study.
\end{abstract}

Key words: stars: flare - Sun: chromosphere - Sun: flares - Sun: photosphere - Sun: transition region - Sun: UV radiation

\section{INTRODUCTION}

The total solar irradiance (TSI) is the energy released by the Sun across the entire electromagnetic spectrum. TSI contributions due to solar flares are normally masked by other solar variations, such as acoustic oscillations and granulation, which occur on timescales similar to the initial rapid bolometric enhancement from solar flares. In the last decade, large solar flares have been detected from the TSI signal directly from the Total Irradiance Monitor (TIM; Kopp et al. 2005b; Kopp \& Lean 2011) and through an epoch superposition technique using the Variability of solar IRradiance and Gravity Oscillations (VIRGO) experiment (Kretzschmar et al. 2010). Solar flares that exhibit measurable visible light enhancements have been deemed "white light flares" (Hudson et al. 1992). It is possible that these white light emissions contribute large amounts of energy to the overall flare energy budget. Their contribution to the TSI energy budget is still an ongoing investigation. This paper aims to help constrain the variation in the TSI due to flares.

Solar flares are the dominant cause of short timescale vacuum ultraviolet (VUV; 0.1-200 nm) emission. VUV wavelengths normally contribute a small portion to the TSI, but can vary by a large factor during solar activity. These emissions are one of the major drivers and energy input source of the Earth's upper atmosphere. The increase in VUV irradiance and charged particles from the Sun can affect Earth's thermosphere, mesosphere, and ionosphere (Qian et al. 2011). Radio transmissions can be interrupted and global positioning systems can be disturbed. An increase in knowledge of the flare VUV and TSI energy release is desired for studies of comparing various energies in solar eruptive events (SEEs), such as was done in Emslie et al. (2012).

Physically, a solar flare is defined as an event where stored magnetic energy is released via an instantaneous (less than a second) rearrangement of stressed magnetic field lines, presumably magnetic reconnection, that accelerates charged particles both toward the solar surface and outward into space (Hudson 2011). The radiative enhancement stems from an increase in particle flux in the solar atmosphere and can be partitioned into two phases, an impulsive phase (IP) and a gradual phase (GP). The initial flare energy release accelerates charged particles inward toward the more dense plasma by the solar surface. This energy input to the lower solar atmosphere heats the plasma and drives an increase of the hard X-ray flux, via bremsstrahlung. The heated particles confined within the magnetic loops subsequently radiate thermally in soft $\mathrm{X}$-rays and in some portions of the VUV.

The initial hard X-ray temporal enhancement is called the IP and the GP is the radiative cooling of the heated plasma in soft X-rays and VUV. The IP generally lasts for 5-10 minutes, and the GP peaks shortly after the IP and can last for several hours. This observed relation between the hard and soft X-ray components, deemed the Neupert effect (Dennis \& Zarro 1993; Veronig et al. 2002; Benz 2008; Hudson 2011), originates from the work of Neupert (1968), who noticed that the integral of the centimeter radio flux roughly matched the soft X-ray flux. The acceleration of electrons outward to relativistic speeds generating radio emission is linked to the hard X-ray emission from the inward-penetrating electrons. Investigations of white light flares in spectral lines and continuum bands with multiple satellites demonstrate their temporal and spatial coherence with hard X-ray emission (Martínez Oliveros et al. 2011; Watanabe et al. 2013). This supports the case of white light emission occurring primarily during the IP.

This study is focused on the radiative aspect of large X-class solar flares. An X-class flare is a flare that has a peak irradiance greater than $10^{-4} \mathrm{~W} \mathrm{~m}^{-2}$ in the $0.1-0.8 \mathrm{~nm}$ channel of the Geostationary Operational Environmental Satellite (GOES) X-Ray Sensor (XRS; García et al. 1994). In this paper, we present results on decomposing flare components into each of the two phases in the VUV and the TSI for the five flares observed by TIM. We also use a VUV empirical model as a proxy for undetected solar flare TSI variations. Our updated algorithm is used to revise values in the study by Emslie et al. (2012). In Section 2, we discuss the data and empirical model used for this study. In Section 3, we describe the time series 
Table 1

TIM Observed Flares (in $10^{30} \mathrm{erg}$ )

\begin{tabular}{lrlccccrrr}
\hline \hline Date & Time & Class & Location & TSI-Tot & TSI-Imp & TSI-Grad & VUV-Tot & VUV-Imp & VUV-Grad \\
\hline 2003 Oct 28 & $9: 51$ & X17 & E08 S16 & 626 & 87 & 539 & 112 & 7.0 & 106 \\
2003 Oct 29 & $20: 37$ & X10 & W10 S17 & 346 & 44 & 302 & 49 & 5.2 & 43 \\
2003 Nov 4 & $19: 29$ & X28 & W83 S19 & 290 & 32 & 258 & 89 & 2.8 & 86 \\
2005 Sep 7 & 17.17 & X17 & E77 S11 & 436 & 6.2 & 430 & 76 & 2.6 & 74 \\
2006 Dec 6 & $18: 29$ & X6.5 & E63 S06 & 76 & 53 & 23 & 20 & 2.1 & 18 \\
\hline
\end{tabular}

Notes. The five X-class flares with detected TIM TSI signatures from solar cycle 23. This list includes total bolometric energy release derived from the TSI time series fits. Also included are the VUV (1-190 nm) energy estimates obtained by integrating the FISM flare profiles. Both energy sets are decomposed into their impulsive and gradual phases.

${ }^{a}$ Designates a limb flare (flare with an east-west position larger than 70).

fitting procedure used to fit the empirical model to the observed TSI data. In Section 4, we explain how these results are used in estimating the total X-class solar flare radiative output during TIM eclipse periods. In Sections 5 and 6, we discuss results and future work, respectively.

\section{DATA}

\subsection{Total Irradiance Monitor}

Total solar irradiance data was obtained from NASA's SOlar Radiation and Climate Experiment (SORCE) mission by the TIM. TIM has been taking solar data since SORCE was launched in 2003 January (Kopp et al. 2005a). This instrument sets the two criteria in determining which flares are to be studied for this analysis: (1) flares that have occurred since 2003 January and (2) flares that have an observed signal response in the TSI. Table 1 lists the five flares that met these criteria. The SORCE spacecraft is in low Earth orbit, which takes about 90 minutes to complete. This orbit leaves TIM with roughly 45 minutes windows of solar observing when not inside Earth's shadow (non-eclipse periods). The TIM has a time cadence of $50 \mathrm{~s}$. The SORCE TIM is the first instrument to have the ability to observe solar flares in the TSI (Kopp et al. 2005b; Kopp \& Lean 2011), partly due to this time cadence. The TIM rigorous calibration and metrology of all its individual components are designed to attain a $100 \mathrm{ppm}(0.010 \%)$ combined standard uncertainty in the TSI (Kopp et al. 2005a). This absolute precision gives TIM the sensitivity necessary to observe solar flares. Each TIM data point has an uncertainty of roughly $\sigma_{\mathrm{TSI}}=2.72 \mathrm{~mW} \mathrm{~m}^{-2}$, corresponding to $2 \mathrm{ppm}\left(1 \mathrm{ppm}=1360 / 10^{6} \mathrm{~W} \mathrm{~m}^{-2}\right)$.

\subsection{Flare Irradiance Spectral Model}

The Flare Irradiance Spectral Model (FISM; Chamberlin et al. 2007, 2008) was used to determine the solar irradiance in the VUV wavelengths from 0.1 to $190 \mathrm{~nm}$. FISM is an empirical model that estimates the solar irradiance in the VUV wavelengths with $60 \mathrm{~s}$ temporal resolution and $1 \mathrm{~nm}$ spectral resolution from 0.1 to $190 \mathrm{~nm}$. These qualities allow the inclusion of variations due to solar flares, solar rotation, and solar cycle variations. FISM utilizes data from the Thermosphere Ionosphere Mesosphere Energetics and Dynamics (TIMED) Solar Extreme-Ultraviolet Experiment (SEE; Woods et al. 2005) and the Upper Atmospheric Research Satellite (UARS) SOlar STellar Irradiance Composition Experiment (SOLSTICE; Rottman et al. 1993). FISM also includes the derived IP and GP for solar flares by using the irradiance and the time derivative from the GOES XRS $0.1-0.8 \mathrm{~nm}$ channel. We analyzed 13 solar flares of solar cycle 23 in the VUV consistent with the Neupert effect (Neupert 1968; Equation (1)). The version of the Neupert effect that we employ takes only the positive values of the soft $\mathrm{X}$-ray flux time derivative, $F_{\mathrm{sxr}}$, which agrees with the hard X-ray flux, $F_{\mathrm{hxr}}$,

$$
\frac{d\left(F_{\mathrm{sxr}}\right)}{d t} \simeq F_{\mathrm{hxr}} .
$$

This relation holds when the magnetic loop plasma is heated by the influx of accelerated particles and cooling by radiation and conduction are negligible (Benz 2008).

\section{DATA ANALYSIS}

The total radiative energy in the VUV wavelengths of each flare observed by GOES XRS can be modeled with FISM. The FISM formulation calculates a daily flux component separately, along with the individual flux contributions from the IP and GP. Therefore, contributions from both the IP and GP are already known and separate in FISM. The fluence (joule $\mathrm{m}^{-2}$ ) for each phase is computed by integrating the irradiance $\left(\mathrm{W} \mathrm{m}^{-2}\right)$ of the IP and GP over their respective temporal durations of the flare and over the VUV wavelengths $(0.1-190 \mathrm{~nm})$ as in Equation (2):

$$
E_{\mathrm{VUV}}=f \int_{t_{\mathrm{start}}}^{t_{\mathrm{end}}} \int_{\lambda_{\min }}^{\lambda_{\max }} I_{\mathrm{FISM}, \mathrm{IP}+\mathrm{GP}}(\lambda, t) d \lambda d t,
$$

where $t_{\text {start }}$ and $t_{\text {end }}$ are the start and end times of each flare phase, respectively. The low and high wavelength bounds are $\lambda_{\min }$ and $\lambda_{\max }$, respectively. The irradiance contributions computed here are solely from the flare enhancement and not from the rest of the Sun. After the fluence is obtained, Equation (3) gives the conversion, $f$, in units of erg $\mathrm{m}^{2}$ joule ${ }^{-1}$, which must be applied to convert from irradiance units of $\mathrm{W} \mathrm{m}^{-2}$ at the Earth to erg at the Sun by multiplying

$$
\begin{aligned}
& f=(1 \mathrm{AU})^{2} 2 \pi \times 10^{7} \\
& f=1.406 \times 10^{30}
\end{aligned}
$$

where $\mathrm{AU}$ is the astronomical unit in meters. This conversion assumes a uniform angular distribution of the flare energy release. A similar calculation was performed in Woods et al. (2006). This algorithm gives an approximation of the energy release in the VUV wavelengths during each phase of the flare with similar accuracies to those of FISM flare estimates. The VUV radiative energies for all flares in this paper are given in Tables 1 and 2.

An amplitude fitting routine employing the FISM model was developed to fit the time profile of flares observed in the TIM TSI to obtain the bolometric radiative energy output due to solar flares. The TIM TSI data was fit to the scaled IP and GP 

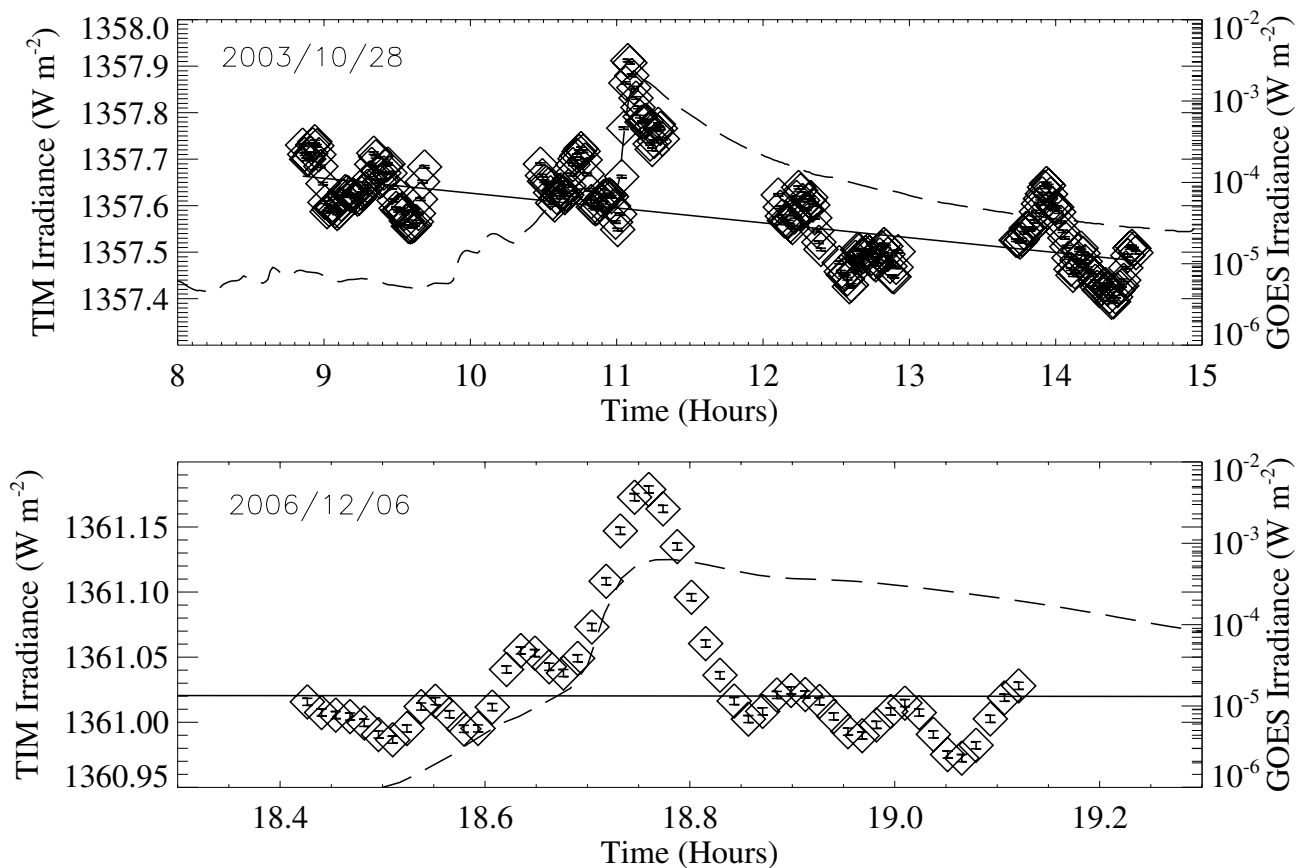

Figure 1. TSI background subtraction for the 2003 October 28 flare and the 2006 December 6 flare that was not in Woods et al. (2006). The solid line is the linear fit to the fluctuating background. The diamonds are the TIM TSI data with error bars from the instrument uncertainties only. The dashed line is the GOES XRS 0.1-0.8 nm, 1 minute time cadence data.

Table 2

TIM Reconstructed Flares (in $10^{30} \mathrm{erg}$ )

\begin{tabular}{lrclcccccc}
\hline \hline Date & Time & Class & Location & TSI-Tot & TSI-Imp & TSI-Grad & VUV-Tot & VUV-Imp & VUV-Grad \\
\hline 2002 Apr 21 & 0:43 & X1.5 & W84 S14 $^{\mathrm{a}}$ & 112 & 3.8 & 108 & 26 & 1.0 & 25 \\
2002 Ju1 23 & $0: 18$ & X4.8 & E72 S13 $^{\mathrm{a}}$ & 102 & 7.2 & 95 & 24 & 1.9 & 22 \\
2003 Nov 2 & $17: 03$ & X8.3 & E56 N14 & 148 & 43 & 105 & 47 & 4.2 & 43 \\
2005 Jan 15 & $0: 22$ & X1.2 & E14 N8 & 8.4 & 2.1 & 6.3 & 2.8 & 0.2 & 2.6 \\
2005 Jan 19 & $8: 03$ & X1.3 & W5 N15 & 64 & 16 & 49 & 22 & 1.5 & 20 \\
2005 Jan 20 & $6: 36$ & X7.1 & W61 N14 & 218 & 40 & 178 & 77 & 3.9 & 73 \\
2006 Dec 5 & $10: 18$ & X 9.0 & E79 S07 & 166 & 19 & 147 & 39 & 5.0 & 34 \\
2006 Dec 13 & $2: 14$ & X3.0 & W23 S05 & 113 & 37 & 76 & 35 & 3.6 & 31 \\
\hline
\end{tabular}

Notes. The eight X-class flares with reconstructed TSI values based on the FISM model flare profiles. The reconstructed TSI impulsive and gradual phases energies are obtained by scaling the VUV impulsive and gradual phase energies as described in Section 4.

a Designates a limb flare (flare with an east-west position larger than 70).

FISM VUV curves to acquire the energy release in each phase. The sum of the energies from each phase gives the total energy from the flare in the TIM TSI. The GOES XRS 0.1-0.8 nm channel was used as a temporal proxy for the GP, and the time derivative of this channel is used as the temporal proxy for the IP. This procedure is the application of the Neupert effect. The TIM TSI flare signal is isolated by subtracting the background TIM TSI irradiance. A linear relationship is found from the irradiance values before and after the flare profile, and the resulting background fit is subtracted off (see Figure 1). To analyze the TIM TSI flare signal, the GOES XRS (from the FISM VUV), IP and GP proxies are scaled such that their sum matches the time signature of the background subtracted TIM TSI data; see Equation (4), Figure 2, and Figure 3. This fit is accomplished by two separate algorithms, an analytical and a numerical one. The subscript, $t$, in the definitions refers to time in the following expression. The analytical algorithm solves for the scaling coefficients of the IP $I_{t}$, with coefficient $a$, and GP $G_{t}$, with coefficient $b$, of the time profiles exactly by a least squares calculation (Bevington \& Robinson 2003; Press et al. 2007) so that the difference between the observed data and model function, $Z_{t}$, is minimized:

$$
Z_{t}=a I_{t}+b G_{t} .
$$

The values obtained by mathematical definition will be the best coefficients if the errors in the data are Gaussian distributed and the errors are relatively small compared to the data value. Our error bars (solely from instrument precision and background subtraction), as displayed in Figures 2 and 3, are small compared to their corresponding values. The data value errors are not necessarily Gaussian distributed, but this merit function still gives a good estimation of the best scale factors.

The values obtained by this methodology can be positive or negative, where negative scale factors for irradiance are nonphysical. A negative scale factor was encountered for the IP of the flare on 2005 September 7, due to a very small IP. In addition to the analytical algorithm, a numerical minimization of chi-square was conducted by the exploration of parameter space along the minimum "bowl" for a predefined grid of values for the $a$ and $b$ coefficients. This method is limited by the choice of gridded values for $a$ and $b$ that are explored. Generally, the analytic and numerical scale factors are consistent with each 

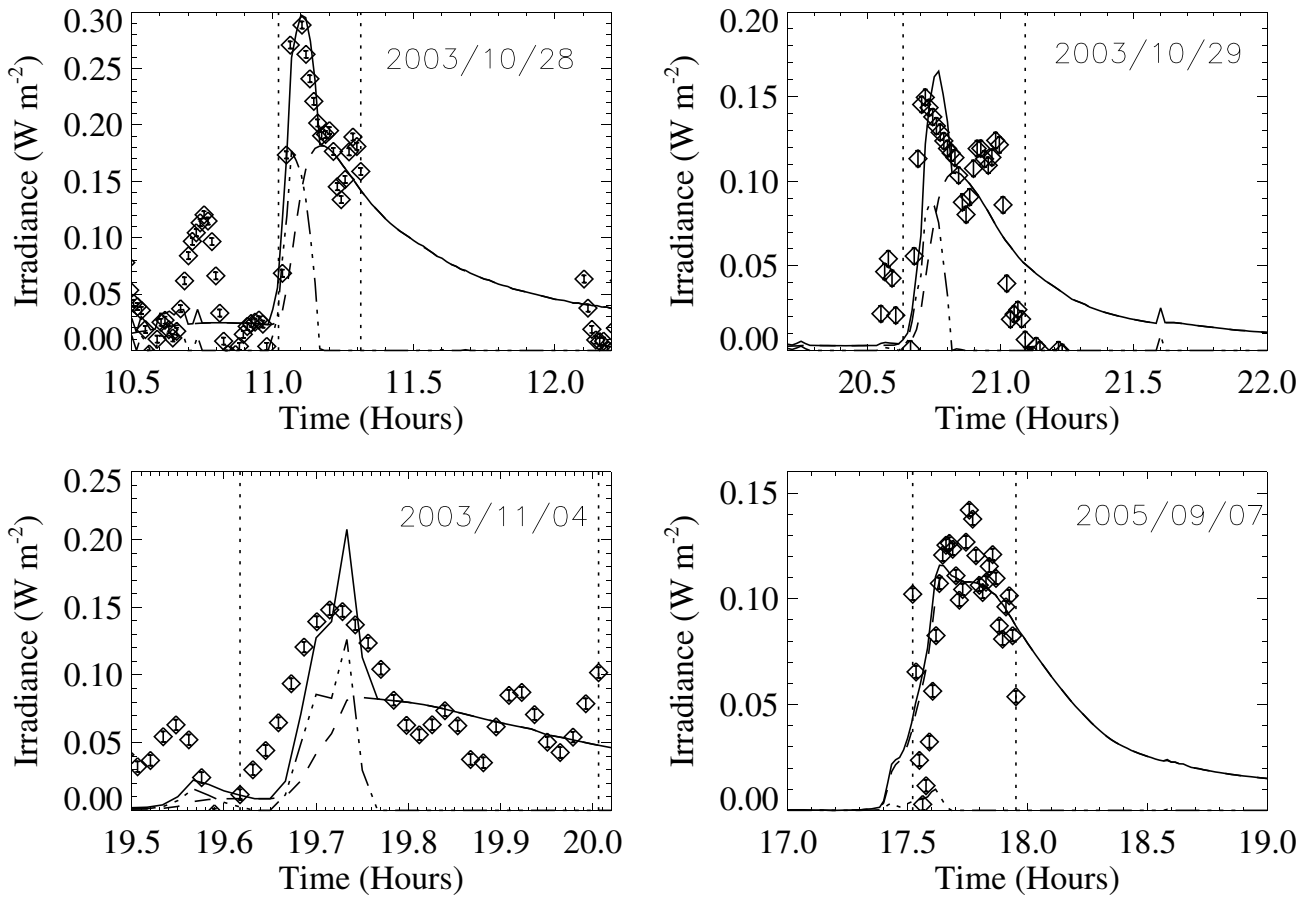

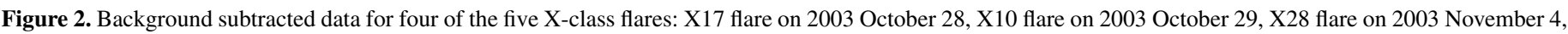

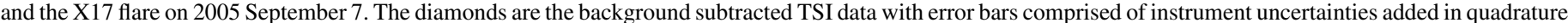

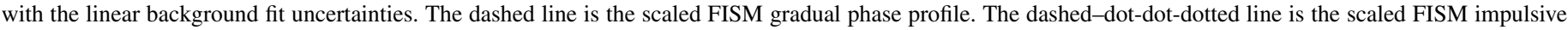

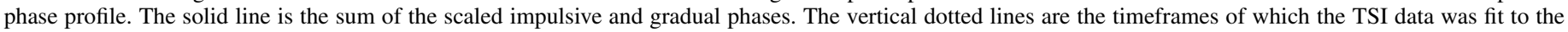
FISM time profiles.

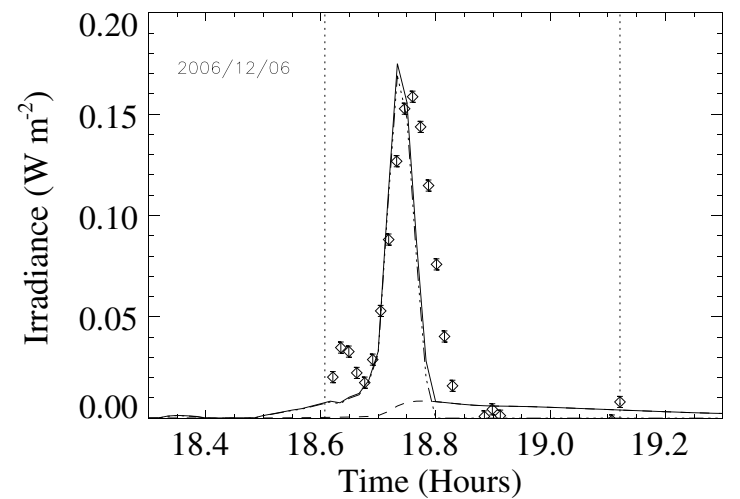

Figure 3. Background subtracted data for the X6.5 flare on 2006 December 6 not included in Woods et al. (2006). The diamonds are the background subtracted TSI data with error bars comprised of instrument uncertainties added in quadrature with the linear background fit uncertainties. The dashed line is the scaled FISM gradual phase profile. The dashed-dot-dot-dotted line is the scaled FISM impulsive phase profile. The solid line is the sum of the scaled impulsive and gradual phases. The vertical dotted lines are the timeframes of which the TSI data was fit to the FISM time profiles.

other. The analytic algorithm for scale factors were used for all flares in this paper, except for the 2005 September 7 flare for the aforementioned reason. The scaled GOES time series were integrated with respect to time, and the conversion factor (Equation (3)) is applied to obtain the TSI contribution for each flare phase at the Sun. The radiative energies for the five flares with an observable TIM TSI signature are given in Table 1.

\section{RECONSTRUCTED TSI FLARE ENERGY RELEASE}

The radiative output from $\mathrm{X}$-class solar flares have large contributions from the VUV wavelength emissions, $\sim 41 \%$ for the GP of center flares and $\sim 23 \%$ for the GP for limb flares. We take limb flares to have an east-west location $>70^{\circ}$. Using the five flares seen in the TIM TSI and estimated VUV emission simultaneously obtained from TIMED SEE and GOES, respectively, the TSI flare irradiances during TIM eclipse periods can be reconstructed. This method can be used to estimate the position-dependent total radiative output from flares, which is described in this section.

The average position-dependent fractional energy contribution to the flare from the VUV to the total radiative output is found for each flare. The inverse of these values are used to scale the VUV energy output for flares not observed due to TIM eclipse periods (when TIM is behind the Earth with respect to the Sun) to obtain an estimation of the total radiative output. This model is still limited by low statistics (five solar flares), but as more large flares occur, more robust algorithms can be developed. The IP $A_{\text {Imp }}$ and the GP $B_{\text {Grad }}$ scale factors for the time-integrated VUV irradiance used to approximate the timeintegrated TSI flare output are given in Equation (5) for the IP and Equation (6) for the GP. The total number of limb or center flares is given by $n$, the index for each individual flare in each category is $i$, the VUV IP energy is VI, the TSI IP energy is $T I$, the VUV GP energy is $V G$, and the TSI GP energy is $T G$ :

$$
\begin{aligned}
A_{\mathrm{Imp}} & =\left(\frac{1}{n} \sum_{i=1}^{n}\left(\frac{V I_{i}}{T I_{i}}\right)\right)^{-1}, \\
B_{\mathrm{Grad}} & =\left(\frac{1}{n} \sum_{i=1}^{n}\left(\frac{V G_{i}}{T G_{i}}\right)\right)^{-1} .
\end{aligned}
$$

These calculations are done separately for center and limb flares. The scale factors for center flares are $A_{\text {Imp }}=10.3$ and $B_{\text {Grad }}=2.4$ for the IP and GP, respectively. Limb flare scale factors obtained were $A_{\mathrm{Imp}}=3.8$ and $B_{\mathrm{Grad}}=4.3$. 


\section{RESULTS AND CONCLUSION}

The TSI energy estimations are the same order of magnitude as previously published values for X-class solar flares $\left(10^{31}-10^{32} \mathrm{erg}\right)$ and have accuracies consistent to the order given by the uncertainties. TSI-radiated energies using the previously described algorithm vary somewhat from the values given in the TIM analysis by Woods et al. (2006) by up to a factor of 1.5 , where an additional flare on 2006 December 6 is included here that occurred after publication of the aforementioned reference. This is believed to be due to a more robust fitting routine for both the IP and GP scaling, but more importantly, to the improved background fitting routine that can easily give a factor of two in the final results due to the long tail in the decay phase that flares have. The latter is most apparent in the 2005 September 7 flare, which is dominated by GP emission.

Our results are also consistent within the $1 \sigma$ uncertainties of the TSI flare contributions derived in the analysis of Kretzschmar et al. (2010) using the PMO and DIRAD radiometers from the VIRGO experiment on the SOlar and Heliospheric Observatory mission (Frohlich et al. 1997). As anticipated, the GP contains the bulk of the energy released in solar flares as compared to the IP (an average of 7.1 times larger), due to the GP lasting much longer. Tables 1 and 2 demonstrate variations in the radiative output of the flares observed at Earth due to center-to-limb variations of optically thick emissions (Worden et al. 2001; Chamberlin et al. 2008). Limb flares have an expected lower energy output because the XUV wavelengths from optically thin coronal emissions dominate the energy release and are unaffected. The optically thick emissions are reduced due to the extended path length through the solar atmosphere. Flares near disk center are more intense than the limb flares in the TSI. This center-to-limb correction can also be applied in reverse to get the TSI radiated output for a limb flare as if it actually occurred on the center of the solar disk. The modeled values are consistent with values given for two flares that occurred before 2003 that were analyzed in Emslie et al. (2004).

This model can be extended for use on flares lower than $\mathrm{X}$-class, which was done in an earlier version of this analysis in the investigation by Emslie et al. (2012). In that study, the reconstructed TSI was computed for the majority of X-class flares of solar cycle 23 that were not observed by TIM due to eclipse periods or flares that occurred before the launch of TIM in 2003. This matured version provides a more accurate estimate of the radiated energy for the energetic comparisons of SEEs. The revised values given here are, in general, larger by roughly a factor of two than those from the Emslie et al. (2012) project due to the modified processes to subtract the highly variable background and, equally as important, the time interval in which the fit is performed and the selection of adequate start and end times for temporal integration of the TSI data.

These newer values are consistent with the conclusions derived in the comprehensive study of Emslie et al. (2012). The bolometric radiated energy is still over an order of magnitude less than the stored non-potential (free) magnetic energy in the studied active regions. This still agrees with the belief that the available magnetic energy is more than sufficient to power the flare components. The bolometric radiated energy is still less than the coronal mass ejection kinetic energy. Moreover, the bolometric radiated energy is comparable to the lower limits of the flare-accelerated particles, where the latter could be up to an order of magnitude greater due to the lower energy cutoff being masked by the thermal plasma radiating in soft $\mathrm{X}$-rays. The conclusion persists that there is sufficient energy in accelerated particles to power the radiative energy release. Finally, the correlation between the soft X-ray plasma and bolometric energy is preserved.

\section{DISCUSSION AND FUTURE WORK}

A full in-depth knowledge of the energy distribution of solar flares across the entire electromagnetic spectrum is difficult to obtain due to lack of simultaneous observations by the instruments at the time of analysis. These modeled results are the best estimations currently achievable, but they are limited to the current TIM TSI data and spectrally to the VUV region. We have broken down the radiative contribution of the VUV (0.1-190 $\mathrm{nm})$ wavelengths to the total radiative energy release for the largest solar flares, partitioned them into two separate phases, and have implemented an empirical model for bolometric flare energy reconstruction. In the future, more steps will be taken to pursue increased knowledge of the spectral energy distribution of flares along with IP and GP composition.

The authors thank the Laboratory for Atmospheric and Space Physics (LASP) for their funding and support, as well as support from the NSF REU program.

\section{APPENDIX}

For completeness, our analytic fitting method is described here. The subscript, $t$, in the definitions refer to time in the following expressions. The analytical algorithm solves for the scaling coefficients of the IP $I_{t}$, with coefficient $a$, and GP $G_{t}$, with coefficient $b$, of the time profiles exactly by a least squares calculation similar to the one in Press et al. (2007). The algorithm is as follows. For a model function, $Z_{t}$, that is linear in the parameters $a$ and $b$ (Equation (A1)):

$$
Z_{t}=a I_{t}+b G_{t} .
$$

The coefficients that give the optimal approximation to the actual observed TSI data, $D_{t}$, with $N$ discrete data points, and propagated uncertainties $\sigma_{t}$, can be found by composing a chi-square merit function, differentiating this function with respect to parameters $a$ and $b$ separately, setting them equal to zero and then solving these two coupled equations for the coefficients $a$ and $b$ (Equations (A2)-(A11)):

$$
\chi^{2}(a, b)=\sum_{t=1}^{N}\left(\frac{D_{t}-a I_{t}-b G_{t}}{\sigma_{t}^{2}}\right)^{2} .
$$

Partial differentiate with respect to $a$ and $b$, set equal to zero for two separate equations:

$$
\begin{gathered}
\frac{\partial\left(\chi^{2}(a, b)\right)}{\partial a}=\sum_{t=1}^{N}-2\left(\frac{D_{t}-a I_{t}-b G_{t}}{\sigma_{t}^{2}}\right) I_{t}=0 \\
\frac{\partial\left(\chi^{2}(a, b)\right)}{\partial b}=\sum_{t=1}^{N}-2\left(\frac{D_{t}-a I_{t}-b G_{t}}{\sigma_{t}^{2}}\right) G_{t}=0 .
\end{gathered}
$$

We use the substitutions,

$$
S_{I I}=\sum_{t=1}^{N}\left(\frac{I_{t}}{\sigma_{t}}\right)^{2}
$$




$$
\begin{gathered}
S_{D I}=\sum_{t=1}^{N} \frac{D_{t} I_{t}}{\sigma_{t}^{2}} \\
S_{I G}=\sum_{t=1}^{N} \frac{I_{t} G_{t}}{\sigma_{t}^{2}} \\
S_{D G}=\sum_{t=1}^{N} \frac{D_{t} G_{t}}{\sigma_{t}^{2}} \\
S_{G G}=\sum_{t=1}^{N}\left(\frac{G_{t}}{\sigma_{t}}\right)^{2}
\end{gathered}
$$

and then solve for $a$ and $b$.

$$
\begin{gathered}
a=\frac{S_{D I} S_{G G}-S_{D G} S_{I G}}{S_{I I} S_{G G}-\left(S_{I G}\right)^{2}} \\
b=\frac{S_{I I} S_{D G}-S_{D I} S_{I G}}{S_{I I} S_{G G}-\left(S_{I G}\right)^{2}} .
\end{gathered}
$$

This gives the impulsive and GP scale factors, $a$ and $b$, respectively.

\section{REFERENCES}

Benz, A. O. 2008, LRSP, 5, 1

Bevington, P. R., \& Robinson, D. K. 2003, Data Reduction and Error Analysis for the Physical Sciences (3rd ed.; New York: McGraw Hill)

Chamberlin, P. C., Woods, T. N., \& Eparvier, F. G. 2007, SpWea, 5, 7005

Chamberlin, P. C., Woods, T. N., \& Eparvier, F. G. 2008, SpWea, 6, 5001

Dennis, B. R., \& Zarro, D. M. 1993, SoPh, 146, 177

Emslie, A. G., Dennis, B. R., Shih, A. Y., et al. 2012, ApJ, 759, 71

Emslie, A. G., Kucharek, H., Dennis, B. R., et al. 2004, JGRA, 109, 10104

Frohlich, C., Andersen, B. N., Appourchaux, T., et al. 1997, SoPh, 170, 1

García, M. A., López, O., Sospedra, J., et al. 1994, AnGeo, 12, 856

Hudson, H. S. 2011, SSRv, 158, 5

Hudson, H. S., Acton, L. W., Hirayama, T., \& Uchida, Y. 1992, PASJ, 44, L77

Kopp, G., Heuerman, K., \& Lawrence, G. 2005a, SoPh, 230, 111

Kopp, G., Lawrence, G., \& Rottman, G. 2005b, SoPh, 230, 129

Kopp, G., \& Lean, J. L. 2011, GeoRL, 38, 1706

Kretzschmar, M., de Wit, T. D., Schmutz, W., et al. 2010, NatPh, 6, 690

Martínez Oliveros, J. C., Couvidat, S., Schou, J., et al. 2011, SoPh, 269, 269

Neupert, W. M. 1968, ApJL, 153, L59

Press, W. H., Teukolsky, S. A., Vetterling, W. T., \& Flannery, B. P. 2007, Numerical Recipes: The Art of Scientific Computing (3rd ed.; New York: Cambridge Univ. Press)

Qian, L., Laštovička, J., Roble, R. G., \& Solomon, S. C. 2011, JGRA, 116, 0 Rottman, G. J., Woods, T. N., \& Sparn, T. P. 1993, JGR, 98, 10667

Veronig, A., Vrsnak, B., Dennis, B. R., et al. 2002, in SOLMAG 2002. Proceedings of the Magnetic Coupling of the Solar Atmosphere Euroconference, ed. H. Sawaya-Lacoste (ESA SSP-505; Noordwijk: ESA), 599

Watanabe, K., Shimizu, T., Masuda, S., Ichimoto, K., \& Ohno, M. 2013, ApJ, 776,123

Woods, T. N., Eparvier, F. G., Bailey, S. M., et al. 2005, JGRA, 110, 1312

Woods, T. N., Kopp, G., \& Chamberlin, P. C. 2006, JGRA, 111, 10

Worden, J. R., Woods, T. N., \& Bowman, K. W. 2001, ApJ, 560, 1020 